\title{
The Gini index and the consistent measurement of inequality among the poor
}

\author{
O. Aristondo ${ }^{1}$ J.L. García-Lapresta ${ }^{2}$ C. Lasso de la Vega ${ }^{3}$ R.A. Marques Pereira ${ }^{4}$ \\ ${ }^{1}$ BRIDGE Research Group, Departamento de Matemática Aplicada, Universidad del País Vasco, Spain. \\ ${ }^{2}$ PRESAD Research Group, Departamento de Economía Aplicada, Universidad de Valladolid, Spain. \\ ${ }^{3}$ BRIDGE Research Group, Departamento de Economía Aplicada IV, Universidad del País Vasco, Spain. \\ ${ }^{4}$ Dipartimento di Informatica e Studi Aziendali, Università degli Studi di Trento, Italy.
}

\begin{abstract}
In several economic fields, such as those related to health, education or poverty, the individuals' characteristics are measured by bounded variables. Accordingly, these characteristics may be indistinctly represented by achievements or shortfalls. A difficulty arises when inequality needs to be assessed. One may focus either on achievements or on shortfalls but the respective inequality rankings may lead to contradictory results. Specifically, this paper concentrates on the poverty measure proposed by Sen. According to this measure the inequality among the poor is captured by the Gini index. However, the rankings obtained by the Gini index applied to either the achievements or the shortfalls do not coincide in general. To overcome this drawback, we show that an OWA operator is underlying in the definition of the Sen measure. The dual decomposition of the OWA operators into a self-dual core and anti-self-dual remainder allows us to propose an inequality component which measures consistently the achievement and shortfall inequality among the poor.
\end{abstract}

Keywords: Aggregation functions, OWA operators, Gini index, achievement and shortfall inequality, dual decomposition.

\section{Introduction}

Poverty reduction is without doubt a goal of development policy in most countries. To evaluate the evolution of poverty over time in some particular region, the differences of poverty across different countries or the effect of different policies in the alleviation of poverty, one should be first able to measure poverty. According to the 1998 Nobel Prize Laureate A.K. Sen [30], any poverty index should be sensitive to the number of people below the poverty line, to the extent of the income shortfall of the poor from the poverty line, and to the exact pattern of the income distribution of the poor. In other words, every poverty measure should be expressed as a function of these three poverty indicators, showing the incidence, the intensity and the inequality of the poverty, respectively. Poverty changes can be more meaningful and easily understandable if poverty indices can be decomposed into these underlying contributing factors. A number of poverty indices and their decompositions have been proposed to explicitly identify these three components. For comprehensive surveys on poverty and inequality measures see [32] and [7]. Besides [8], [27], [34] and [1], some of them may be found in [21].

As regards the inequality component, Sen [30] points out that "a transfer of income from a person below the poverty line to anyone who is richer must increase the poverty measure". All the inequality indices satisfy the Pigou-Dalton principle, which is considered the basic axiom of inequality measurement. This principle establishes that a transfer of income from a rich individual to a poorer one decreases inequality as long as the poor individual does not become richer than the rich one.

Since a transfer of income from a poorer to a richer person entails a transfer of the shortfall from the latter to the former, the poverty measure is bound to decrease if the inequality component involved in the index is defined in terms of either incomes or shortfalls. In fact, in the mentioned decompositions this third component refers to income inequality or to shortfall inequality indistinctly. For instance, whereas in the original proposals of [30] and [31] the "Gini index of the poor income" takes part in the decompositions, [27] and [34] derive alternative decompositions in which the "Gini index of the gaps" is included. Similarly, the "inequality among the poor" is captured in terms of gaps in the TIP curves introduced by [20] and in the decomposition for the FGT indices ([14]) proposed by [1]. However, as will be shown below, the choice between income and shortfall inequality is not innocuous and different choices may lead to contradictory results. This difficulty arises not only in poverty measurement but also in different economic fields in which bounded variables are involved. Recent papers (among them [9], [12] and [23]) deal with this issue in health measurement. The results derived by [23] may have a straightforward application to the measurement of the inequality among the poor. They introduce a property of consistency which requires that achievement and shortfall in- 
equality rankings should not be reversed, and show that all relative and intermediate inequality indices fail their requirement. Accordingly, whenever a relative or intermediate inequality index is involved in the decomposition of a poverty index, the inequality component is not consistent. We think this is a serious drawback which may distort the conclusions in the analysis of the poverty trends and, consequently, the poverty decompositions are found wanting in displaying changes in the inequality among the poor, one of their main points.

This paper concentrates on the Sen index [30]. Two different decompositions of this index have been proposed ([30] and [34]). The inequality among the poor is captured by the Gini index, applied either to the poor income or to the shortfall of the poor. However, as shown in [23], no relative inequality index offers consistent results.

In this paper a different point of view is proposed. We show that the Sen poverty index may be interpreted as an OWA operator ([37]). Consequently, the dual decomposition of aggregation functions into a self-dual core and anti-self-dual remainder proposed by [17] may be used. We show that these two terms can be interpreted as measures of the intensity and the inequality among the poor, respectively. The anti-self-duality of the remainder component guarantees that inequality among the poor does not change if one focus either on incomes or on shortfalls. These inequality components will allow policy makers to determine in a consistent way if inequality among the poor has increased or decreased.

\section{Aggregation functions}

In this section we present notation and basic definitions regarding aggregation functions on $I^{n}$, where either $I=[0,1]$ or $I=[0, \infty)$, with $n \in \mathbb{N}$ and $n \geq 2$ throughout the text.

Notation. Points in $I^{n}$ will be denoted by means of boldface characters: $\boldsymbol{x}=\left(x_{1}, \ldots, x_{n}\right), \mathbf{1}=(1, \ldots, 1), \quad \mathbf{0}=(0, \ldots, 0)$ and, consequently, for every $x \in I$ we have $x \cdot \mathbf{1}=(x, \ldots, x)$. Given $\boldsymbol{x}, \boldsymbol{y} \in I^{n}$, by $\boldsymbol{x} \geq \boldsymbol{y}$ we mean $x_{i} \geq y_{i}$ for every $i \in\{1, \ldots, n\}$; by $\boldsymbol{x}>\boldsymbol{y}$ we mean $\boldsymbol{x} \geq \boldsymbol{y}$ and $\boldsymbol{x} \neq \boldsymbol{y}$. Given $\boldsymbol{x} \in I^{n}$, with $\left(x_{(1)}, \ldots, x_{(n)}\right)$ we denote the increasing ordered version of $\boldsymbol{x}$, i.e., $x_{(i)}$ is the $i$-th lowest number of $\left\{x_{1}, \ldots, x_{n}\right\}$. Moreover, $x_{(1)}=\min \left\{x_{1}, \ldots, x_{n}\right\}$ and $x_{(n)}=\max \left\{x_{1}, \ldots, x_{n}\right\}$. On the other hand, $\mu$ will denote the arithmetic mean, i.e., $\mu(\boldsymbol{x})=\left(x_{1}+\cdots+x_{n}\right) / n$. Given a permutation on $\{1, \ldots, n\}$, i.e., a bijection $\sigma:\{1, \ldots, n\} \longrightarrow\{1, \ldots, n\}$, with $\boldsymbol{x}_{\sigma}$ we denote $\left(x_{\sigma(1)}, \ldots, x_{\sigma(n)}\right)$.

We begin by defining standard properties of real functions on $I^{n}$. For further details the interested reader is referred to [13], [6], [3], [17] and [19].
Definition 1 Let $A: I^{n} \longrightarrow \mathbb{R}$ be a function.

1. $A$ is idempotent if for every $x \in I: A(x \cdot \mathbf{1})=x$.

2. $A$ is symmetric if for every permutation $\sigma$ on $\{1, \ldots, n\}$ and every $\boldsymbol{x} \in I^{n}: A\left(\boldsymbol{x}_{\sigma}\right)=A(\boldsymbol{x})$.

3. $A$ is monotonic if for all $\boldsymbol{x}, \boldsymbol{y} \in I^{n}: \boldsymbol{x} \geq \boldsymbol{y} \Rightarrow$ $A(\boldsymbol{x}) \geq A(\boldsymbol{y})$.

4. $A$ is strictly monotonic if for all $\boldsymbol{x}, \boldsymbol{y} \in I^{n}$ : $\boldsymbol{x}>\boldsymbol{y} \Rightarrow A(\boldsymbol{x})>A(\boldsymbol{y})$.

5. A is compensative if for every $\boldsymbol{x} \in I^{n}: x_{(1)} \leq$ $A(\boldsymbol{x}) \leq x_{(n)}$.

6. $A$ is self-dual if $I=[0,1]$ and for every $\boldsymbol{x} \in$ $[0,1]^{n}: A(\mathbf{1}-\boldsymbol{x})=1-A(\boldsymbol{x})$.

7. $A$ is anti-self-dual if $I=[0,1]$ and for every $\boldsymbol{x} \in[0,1]^{n}: A(\mathbf{1}-\boldsymbol{x})=A(\boldsymbol{x})$.

8. $A$ is invariant for translations if for all $t \in \mathbb{R}$ and $\boldsymbol{x} \in I^{n}: A(\boldsymbol{x}+t \cdot \mathbf{1})=A(\boldsymbol{x})$ whenever $\boldsymbol{x}+t \cdot \mathbf{1} \in I^{n}$.

9. $A$ is stable for translations if for all $t \in \mathbb{R}$ and $\boldsymbol{x} \in I^{n}: A(\boldsymbol{x}+t \cdot \mathbf{1})=A(\boldsymbol{x})+t$ whenever $\boldsymbol{x}+t \cdot \mathbf{1} \in I^{n}$.

10. $A$ is scale invariant (or homothetic) if for all $\lambda>0$ and $\boldsymbol{x} \in I^{n}: A(\lambda \cdot \boldsymbol{x})=\lambda \cdot A(\boldsymbol{x})$ whenever $\lambda \cdot \boldsymbol{x} \in I^{n}$.

Definition 2 Let $\left\{A^{(k)}\right\}_{k \in \mathbb{N}}$ be a sequence of functions, with $A^{(k)}: I^{k} \longrightarrow \mathbb{R}$ and $A^{(1)}(x)=x$ for every $x \in I . \quad\left\{A^{(k)}\right\}_{k \in \mathbb{N}}$ is invariant for replications if for all $\boldsymbol{x} \in I^{n}$ and any number of replications $m \in \mathbb{N}$ of $\boldsymbol{x}$ :

$$
A^{(m n)}(\overbrace{\boldsymbol{x}, \ldots, \boldsymbol{x}}^{m})=A^{(n)}(\boldsymbol{x}) .
$$

Definition 3 Consider the binary relation $\succcurlyeq$ on $I^{n}$ defined as

$$
\boldsymbol{x} \succcurlyeq \boldsymbol{y} \Leftrightarrow \sum_{i=1}^{n} x_{i}=\sum_{i=1}^{n} y_{i} \text { and } \sum_{i=1}^{k} x_{(i)} \leq \sum_{i=1}^{k} y_{(i)},
$$

for every $k \in\{1, \ldots, n-1\}$.

1. $A: I^{n} \longrightarrow I$ is Schur-convex or S-convex if for all $\boldsymbol{x}, \boldsymbol{y} \in I^{n}$ :

$$
\boldsymbol{x} \succcurlyeq \boldsymbol{y} \Rightarrow A(\boldsymbol{x}) \geq A(\boldsymbol{y})
$$

2. $A: I^{n} \longrightarrow I$ is Schur-concave or S-concave if for all $\boldsymbol{x}, \boldsymbol{y} \in I^{n}$ :

$$
\boldsymbol{x} \succcurlyeq \boldsymbol{y} \Rightarrow A(\boldsymbol{x}) \leq A(\boldsymbol{y})
$$

Definition 4 Given $\boldsymbol{x}, \boldsymbol{y} \in I^{n}$, we say that $\boldsymbol{y}$ is obtained from $\boldsymbol{x}$ by a progressive transfer if there exist two individuals $i, j \in\{1, \ldots, n\}$ and $h>0$ such that $x_{i}<x_{j}, y_{i}=x_{i}+h \leq x_{j}-h=y_{j}$ and $y_{k}=x_{k}$ for every $k \in\{1, \ldots, n\} \backslash\{i, j\}$.

A classical result (see [25, Ch. 4, Prop. A.1]) establishes that $\boldsymbol{x} \succcurlyeq \boldsymbol{y}$ if and only if $\boldsymbol{y}$ can be derived from $\boldsymbol{x}$ by means of a finite sequence of permutations and/or progressive transfers. 
Definition 5 A function $A: I^{n} \longrightarrow I$ is called an $n$-ary averaging aggregation function (AAF) if it is idempotent and monotonic. An AAF is said to be strict if it is strictly monotonic.

For the sake of simplicity, the $n$-arity is omitted whenever it is clear from the context.

It is easy to see that every AAF is compensative. Anti-self-duality and invariance for translations are incompatible with idempotency, one of the defining properties of AAFs. Nevertheless, anti-self-duality and invariance for translations play an important role in this paper.

\section{Dual decomposition}

In this section we briefly recall the so-called dual decomposition of an AAF into its self-dual core and associated anti-self-dual remainder, due to [17] (a generalization of that dual decomposition can be found in [24]). First we introduce the concepts of self-dual core and anti-self-dual remainder of an AAF, establishing which properties are inherited in each case from the original AAF.

Definition 6 Let $A:[0,1]^{n} \longrightarrow[0,1]$ be an $A A F$. The AAF $A^{*}:[0,1]^{n} \longrightarrow[0,1]$ defined as

$$
A^{*}(\boldsymbol{x})=1-A(\mathbf{1}-\boldsymbol{x})
$$

is known as the dual of $A$.

Clearly, $\left(A^{*}\right)^{*}=A$, which means that dualization is an involution. An AAF $A$ is self-dual if and only if $A^{*}=A$.

\subsection{The self-dual core}

Aggregation functions are not in general self-dual. However, a self-dual AAF can be associated to any AAF in a simple manner. The construction of the so-called self-dual core of an AAF $A$ is as follows.

Definition 7 Let $A:[0,1]^{n} \longrightarrow[0,1]$ be an $A A F$. The function $\widehat{A}:[0,1]^{n} \longrightarrow[0,1]$ defined as

$$
\widehat{A}(\boldsymbol{x})=\frac{A(\boldsymbol{x})+A^{*}(\boldsymbol{x})}{2}=\frac{A(\boldsymbol{x})-A(\mathbf{1}-\boldsymbol{x})+1}{2}
$$

is called the core of $A$.

Since $\widehat{A}$ is self-dual, we say that $\widehat{A}$ is the selfdual core of the AAF $A$. Notice that $\widehat{A}$ is clearly an AAF.

The following results (excepting that invariance for replications is inherited by the core; the proof is immediate) can be found in [17].

Proposition 1 Let $A:[0,1]^{n} \longrightarrow[0,1]$ be an $A A F . A$ is self-dual if and only if $\widehat{A}(\boldsymbol{x})=A(\boldsymbol{x})$ for every $\boldsymbol{x} \in[0,1]^{n}$.

Proposition 2 The self-dual core $\widehat{A}$ inherits from $A$ the properties of continuity, idempotency (hence, compensativeness), symmetry, strict monotonicity, stability for translations, and invariance for replications, whenever $A$ has these properties.

\subsection{The anti-self-dual remainder}

We now introduce the anti-self-dual remainder $\widetilde{A}$, which is simply the difference between the original AAF $A$ and its self-dual core $\widehat{A}$.

Definition 8 Let $A:[0,1]^{n} \longrightarrow[0,1]$ be an $A A F$. The function $\widetilde{A}:[0,1]^{n} \longrightarrow \mathbb{R}$ defined as $\widetilde{A}(\boldsymbol{x})=$ $A(\boldsymbol{x})-\widehat{A}(\boldsymbol{x})$, that is

$$
\widetilde{A}(\boldsymbol{x})=\frac{A(\boldsymbol{x})-A^{*}(\boldsymbol{x})}{2}=\frac{A(\boldsymbol{x})+A(\mathbf{1}-\boldsymbol{x})-1}{2},
$$

is called the remainder of $A$.

Since $\widetilde{A}$ is anti-self-dual, we say that $\widetilde{A}$ is the anti-self-dual remainder of the AAF $A$. Clearly, $\widetilde{A}$ is not an AAF. In particular, $\widetilde{A}(\mathbf{0})=\widetilde{A}(\mathbf{1})=0$ violates idempotency and implies that $\widetilde{A}$ is either non monotonic or everywhere null. Moreover, $-0.5 \leq$ $\widetilde{A}(\boldsymbol{x}) \leq 0.5$ for every $\boldsymbol{x} \in[0,1]^{n}$.

The following results can be found in [17] (excepting that invariance for replications is inherited by the remainder - the proof is immediate- and that $\mathrm{S}$ convexity and S-concavity are also inherited by the remainder).

Proposition 3 Let $A:[0,1]^{n} \longrightarrow[0,1]$ be an $A A F$. $A$ is self-dual if and only if $\widetilde{A}(\boldsymbol{x})=0$ for every $\boldsymbol{x} \in[0,1]^{n}$.

Proposition 4 The anti-self-dual remainder $\widetilde{A} i n$ herits from $A$ the properties of continuity, symmetry, invariance for replications, plus also $S$ convexity and S-concavity, whenever $A$ has these properties.

Summarizing, every AAF $A$ decomposes additively $A=\widehat{A}+\widetilde{A}$ in two components: the self-dual core $\widehat{A}$ and the anti-self-dual remainder $\widetilde{A}$, where only $\widehat{A}$ is an AAF.

The following result concerns two more properties of the anti-self-dual remainder based directly on the definition $\widetilde{A}=A-\widehat{A}$ and the corresponding properties of the self-dual core (see [17]).

Proposition 5 Let $A:[0,1]^{n} \longrightarrow[0,1]$ be an $A A F$.

1. $\widetilde{A}(x \cdot \mathbf{1})=0$ for every $x \in[0,1]$.

2. If $A$ is stable for translations, then $\widetilde{A}$ is invariant for translations.

These properties of the anti-self-dual remainder are suggestive. The first statement establish that anti-self-dual remainders are null on the main diagonal. The second statement applies to the subclass of stable AAFs. In such case, self-dual cores are stable and therefore anti-self-dual remainders are invariant for translations. In other words, if the AAF $A$ is stable for translations, the value $\widetilde{A}(\boldsymbol{x})$ does not depend on the average value of the $\boldsymbol{x}$ coordinates, but only on their numerical deviations from that average value. These properties of the anti-self-dual remainder $\widetilde{A}$ suggest that it may give some indication on the dispersion of the $\boldsymbol{x}$ coordinates. 


\section{OWA operators}

In 1988 Yager [37] introduced OWA operators as a tool for aggregating numerical values in multicriteria decision making. An OWA operator is similar to a weighted mean, but with the values of the variables previously ordered in a decreasing way. Because of these properties, OWA operators have been widely used in the literature (see, for instance, [38] and [39]).

Definition 9 Given a weighting vector $\boldsymbol{w}=$ $\left(w_{1}, \ldots, w_{n}\right) \in[0,1]^{n}$ satisfying $\sum_{i=1}^{n} w_{i}=1$, the OWA operator associated with $\boldsymbol{w}$ is the $A A F$ $A_{w}: I^{n} \longrightarrow I$ defined as

$$
A_{w}(\boldsymbol{x})=\sum_{i=1}^{n} w_{i} x_{\sigma(i)}
$$

where $\sigma$ is a permutation of $\{1, \ldots, n\}$ such that $x_{\sigma(1)} \geq \cdots \geq x_{\sigma(n)}$.

OWA operators are continuous, idempotent (hence, compensative), symmetric, and stable for translations. Moreover, an OWA operator $A_{w}$ is self-dual if and only if $w_{n+1-i}=w_{i}$ for every $i \in\{1, \ldots, n\}$ (see [16, Proposition 5]).

In general, the self-dual core $\widehat{A}_{w}$ and the anti-selfdual remainder $\widetilde{A}_{w}$ of an OWA operator $A_{w}$ can be written as

$$
\begin{aligned}
& \widehat{A}_{w}(\boldsymbol{x})=\sum_{i=1}^{n} \frac{w_{i}+w_{n-i+1}}{2} x_{\sigma(i)} \\
& \widetilde{A}_{w}(\boldsymbol{x})=\sum_{i=1}^{n} \frac{w_{i}-w_{n-i+1}}{2} x_{\sigma(i)} .
\end{aligned}
$$

As we know, the self-dual core $\widehat{A}_{w}$ is an AAF. Moreover, since $\sum_{i=1}^{n}\left(w_{i}+w_{n-i+1}\right) / 2=1$, the selfdual core $\widehat{A}_{w}$ is again an OWA operator, that is $\widehat{A}_{w}=A_{\widehat{w}}$ with $\widehat{w}_{i}=\left(w_{i}+w_{n-i+1}\right) / 2$ for every $i \in\{1, \ldots, n\}$.

On the other hand, the anti-self-dual remainder $\widetilde{A}_{w}$ is not an AAF. Notice, in particular, that $\widetilde{A}_{w}(\mathbf{1})=\sum_{i=1}^{n}\left(w_{i}-w_{n-i+1}\right) / 2=0$.

The self-dual core and the anti-self-dual remainder can be equivalently written as follows

$$
\begin{aligned}
& \widehat{A}_{w}(\boldsymbol{x})=\sum_{i=1}^{n} w_{i} \frac{x_{\sigma(i)}+x_{\sigma(n-i+1)}}{2} \\
& \widetilde{A}_{w}(\boldsymbol{x})=\sum_{i=1}^{n} w_{i} \frac{x_{\sigma(i)}-x_{\sigma(n-i+1)}}{2} .
\end{aligned}
$$

These expressions show clearly that the self-dual core is a weighted average of pairwise averages of $\boldsymbol{x}$ coordinates, whereas the anti-self-dual remainder is a weighted average of pairwise differences of $\boldsymbol{x}$ coordinates. The anti-self-dual remainder is therefore independent of the overall average of the coordinates of $\boldsymbol{x}$ and constitutes a form of dispersion measure. Moreover, it is straightforward to prove that $w_{1} \geq \cdots \geq w_{n}$ implies $\widetilde{A}_{w}(\boldsymbol{x}) \geq 0$ and $w_{1} \leq \cdots \leq w_{n}$ implies $\widetilde{A}_{w}(\boldsymbol{x}) \leq 0$.

\section{Generalized Gini welfare functions}

We consider a population consisting of $n$ individuals, with $n \geq 2$. An income distribution is represented by a vector $\boldsymbol{x}=\left(x_{1}, \ldots, x_{n}\right) \in[0, \infty)^{n}$, where $x_{i}$ is the income of individual $i$.

Definition $10 A$ welfare function is a nonconstant function $W:[0, \infty)^{n} \longrightarrow \mathbb{R}$ that is continuous, strictly $S$-concave and monotonic.

Definition 11 Given a weighting vector $\boldsymbol{w}=$ $\left(w_{1}, \ldots, w_{n}\right) \in[0,1]^{n}$ satisfying $w_{1} \geq \cdots \geq w_{n} \geq 0$ and $\sum_{i=1}^{n} w_{i}=1$, the generalized Gini welfare function (or rank dependent general welfare function) associated with $\boldsymbol{w}$ is the $A A F W_{w}:[0, \infty)^{n} \longrightarrow \mathbb{R}$ defined as

$$
W_{w}(\boldsymbol{x})=\sum_{i=1}^{n} w_{i} x_{(i)}
$$
$36]$.

On this, see [4], [10, 11], [26], [28], [33] and [35,

Notice that generalized Gini welfare functions are OWA operators:

$$
W_{w}(\boldsymbol{x})=\sum_{i=1}^{n} w_{i}^{\prime} x_{\sigma(i)},
$$

where $\sigma$ is a permutation of $\{1, \ldots, n\}$ such that $x_{\sigma(1)} \geq \cdots \geq x_{\sigma(n)}$ and $w_{i}^{\prime}=w_{n-i+1}$.

Positivity of $w_{i}$ guarantees that $W_{w}$ satisfies the Pareto Principle, that is, it is increasing in $x_{i}$. Decreasingness of the sequence of coefficients is necessary and sufficient for S-concavity of $W_{w}$. All the functions in (1) are both, stable for translations and scale invariant.

Definition $12 A n$ inequality index is a nonconstant function $I:[0, \infty)^{n} \longrightarrow \mathbb{R}$ that is continuous and strictly S-convex. The inequality index is relative if $I$ is scale invariant and absolute whenever I is invariant for translations.

Following the conventional approach ([22], [2], [29] or [5], among others) any welfare function in (1) may be used to derive both relative and absolute inequality indices as follows

$$
\begin{gathered}
I_{R}(\boldsymbol{x})=1-\frac{W_{w}(\boldsymbol{x})}{\mu(\boldsymbol{x})} \\
I_{A}(\boldsymbol{x})=\mu(\boldsymbol{x})-W_{w}(\boldsymbol{x}) .
\end{gathered}
$$

The Gini welfare function appears taking in (1)

$$
w_{i}=\frac{2(n-i)+1}{n^{2}} .
$$

With these weights, equation (2) becomes the Gini index

$$
G(\boldsymbol{x})=\frac{1}{n^{2} \mu(\boldsymbol{x})} \sum_{i=1}^{n}(2(n-i)+1)\left(\mu(\boldsymbol{x})-x_{(i)}\right),
$$


whereas equation (3) is referred to as the absolute Gini index

$$
G_{A}(\boldsymbol{x})=\frac{1}{n^{2}} \sum_{i=1}^{n}(2(n-i)+1)\left(\mu(\boldsymbol{x})-x_{(i)}\right) .
$$

The Gini index [18] is the most commonly used measure of inequality. This index varies between 0 , which reflects complete equality and 1, which indicates complete inequality. The Gini index is relative and invariant for replications, which allows inequality comparisons between societies with different populations.

In contrast with other inequality measures, the Gini index has a number of desirable properties. First, it easily accommodates non-positive incomes. Secondly, inequality as measured by this index depends on the significance of the income gaps in society, and finally, the simple relationship with the Gini absolute index given by $G_{A}(\boldsymbol{x})=\mu(\boldsymbol{x}) G(\boldsymbol{x})$.

\section{Poverty measures}

First, this section briefly summarizes some basic notions about poverty measures. With respect to the notation and definitions we follow [15].

Since [30], any poverty measure consists of a method to identify the poor together with an aggregative measure. Thus, the first step to define a poverty measure is the identification of the poor people in society. This step requires the specification of a poverty line $z \in(0, \infty)$ which represents the necessary income to maintain a minimum level of living. For an income distribution $\boldsymbol{x}$, person $i$ is considered to be poor if $x_{i}<z$. Otherwise the person is non-poor or rich.

We denote the set of poor people by

$$
Q(\boldsymbol{x}, z)=\left\{i \in\{1, \ldots, n\} \mid x_{i}<z\right\}
$$

and by $q(\boldsymbol{x}, z)$ the number of the poor, i.e., $q(\boldsymbol{x}, z)=\# Q(\boldsymbol{x}, z)$.

Once the poor people have been identified, the second step to determine the extent of poverty involves the aggregation scheme. In what follows, a poverty measure is a non-constant function $P(\boldsymbol{x}, z)$ of the income distribution $\boldsymbol{x}$ and the poverty line $z$.

\subsection{Axioms}

Some axioms are usually assumed for a poverty measure.

- Poverty Focus (PF): For all $\boldsymbol{x}, \boldsymbol{y} \in[0, \infty)^{n}$ and $z \in(0, \infty)$, if $Q(\boldsymbol{x}, z)=Q(\boldsymbol{y}, z)=Q$ and $x_{i}=y_{i}$ for every $i \in Q$, then $P(\boldsymbol{x}, z)=$ $P(\boldsymbol{y}, z)$.

- Poverty Monotonicity (PM): For all $\boldsymbol{x}, \boldsymbol{y} \in$ $[0, \infty)^{n}$ and $z \in(0, \infty)$, if $Q(\boldsymbol{x}, z)=Q(\boldsymbol{y}, z)=$ $Q$ and $\boldsymbol{x}=\boldsymbol{y}$ except for $x_{i}>y_{i}$ with $i \in Q$, then $P(\boldsymbol{x}, z)<P(\boldsymbol{y}, z)$.
Since poverty measurement is concerned with the deprivations of poor people, these two properties, postulated by Sen [30], are considered as the basic axioms for a poverty measure. Thus, axiom $\mathbf{P F}$ requires that a poverty index should not depend on the income of the non-poor people, i.e., the poverty level should not vary if the rich incomes change, as long as the set of poor people remains unchanged. On the other hand, axiom PM demands that poverty should increase if the income of a poor person decreases.

The following axiom is concerned with inequality among the poor. In the inequality field, the Pigou-Dalton transfer principle establishes that a progressive transfer, that is a transfer from a richer person to a poorer one, should decrease inequality. Accordingly, a progressive transfer among the poor should decrease inequality among the poor. Sen [30] introduces the counterpart of this principle in the poverty field, requiring poverty also to decrease. This is captured by the following axiom.

- Transfer Sensitivity (TS): For all $\boldsymbol{x}, \boldsymbol{y} \in$ $[0, \infty)^{n}$ and $z \in(0, \infty)$, if $\boldsymbol{y}$ is obtained from $\boldsymbol{x}$ by a progressive transfer among the poor, then $P(\boldsymbol{y}, z)<P(\boldsymbol{x}, z)$.

A progressive transfer among the poor entails an increment of income for one poor individual, and a decrement for another poor person, the richer of the two. This TS axiom goes beyond PM and demands that greater weight should be placed on the poorer person and that poverty should decrease if inequality among the poor decreases.

A normalization condition is also usually assumed in the poverty measurement. This property requires that if all the individuals are non-poor, then the society deprivation level is equal to 0 .

- Normalization $(\mathbf{N})$ : For all $\boldsymbol{x}, \boldsymbol{y} \in[0, \infty)^{n}$ and $z \in(0, \infty), P(\boldsymbol{x}, z)=0$ if and only if $Q(\boldsymbol{x}, z)=\emptyset$, that is $x_{i} \geq z$ for every $i \in\{1, \ldots, n\}$.

The two following invariance axioms are also standard requirements for a poverty measure:

- Poverty Symmetry (PS): For all $\boldsymbol{x} \in[0, \infty)^{n}$, $z \in(0, \infty)$, and permutation $\sigma$ on $\{1, \ldots, n\}$, it holds that $P\left(\boldsymbol{x}_{\sigma}, z\right)=P(\boldsymbol{x}, z)$.

- Replication Invariance (RI): For all $\boldsymbol{x} \in$ $[0, \infty)^{n}$ and $z \in(0, \infty)$, if $\boldsymbol{y}$ is obtained from $\boldsymbol{x}$ by a replication, that is $\boldsymbol{y}=(\overbrace{\boldsymbol{x}, \ldots, \boldsymbol{x}}^{m})$ for some $m \in \mathbb{N}$, then $P(\boldsymbol{y}, z)=P(\boldsymbol{x}, z)$.

PS establishes that no other characteristic apart from the income deprivation matters in defining a poverty index. In turn, RI allows us to compare populations of different sizes.

The first poverty measure introduced in the literature has been the headcount ratio

$$
H:[0, \infty)^{n} \times(0, \infty) \longrightarrow[0,1]
$$


defined as

$$
H(\boldsymbol{x}, z)=\frac{q(\boldsymbol{x}, z)}{n},
$$

which measures the percentage of poor people in the society. This is a crude index, which is able to capture the incidence of poverty. However, it is able to capture neither the intensity nor the inequality among the poor. In fact it violates both PM and TS, since it does not change if the income of a poor decreases, and under progressive transfers among the poor.

In most cases, measuring poverty involves gauging the extent of the deprivation felt by each individual, once the income poverty line has been determined. One of the most used procedures to measure individual $i$ 's shortfall is to consider the normalized gap of individual $i$.

Definition 13 For all $\boldsymbol{x} \in[0, \infty)^{n}$ and $z \in$ $(0, \infty)$, the normalized gap of individual $i$ is defined as

$$
g_{i}=\max \left\{\frac{z-x_{i}}{z}, 0\right\} .
$$

Notice that $g_{i} \in[0,1], g_{i}=0 \Leftrightarrow x_{i} \geq z$, and $g_{i}=1 \Leftrightarrow x_{i}=0$.

Moreover, the normalized gaps are invariant under proportional income changes. In other words, the function $\mathbb{G}:[0, \infty)^{n} \times(0, \infty) \longrightarrow[0,1]^{n}$ defined by $\mathbb{G}(\boldsymbol{x}, z)=\left(g_{1}, \ldots, g_{n}\right)$ is homogeneous of degree 0: $\mathbb{G}\left(\lambda x_{i}, \ldots, \lambda x_{n}, \lambda z\right)=\mathbb{G}\left(x_{i}, \ldots, x_{n}, z\right)$, for every $\lambda>0$.

On the other hand, a progressive transfer among the poor people lead to an increment in the richer individual gap whereas the poorer person gap decreases. Since the richer gap is smaller than the poorer one, then the progressive transfers among the poor incomes are equivalent to the progressive transfers among the poor gaps. Then, according to $[25$, Ch. 4 , Prop. A.1], the TS axiom is to be fulfilled whenever the function is S-convex either in incomes or in gaps.

Definition 14 The mean among the poor of the normalized poverty gaps is given by the function $M:[0, \infty)^{n} \times(0, \infty) \longrightarrow[0,1]$ defined as

$$
M(\boldsymbol{x}, z)=\mu\left(\boldsymbol{g}_{p}\right)=\frac{1}{q} \sum_{i \in Q} g_{i},
$$

where $\boldsymbol{g}_{p}$ is the vector whose components are the positive normalized poverty gaps generated by $\boldsymbol{x}$ and $z$. $M(\boldsymbol{x}, z)$ is called the aggregate income gap ratio.

This index usually measures the intensity of poverty and gives the minimum cost of eliminating poverty but does not reflect the inequality among the poor.

The generalized Gini welfare functions in equation (1) can be also used to aggregate the poor gaps. Given $\boldsymbol{x} \in[0, \infty)^{n}$, consider again $\boldsymbol{g}_{p}$ the vector whose components are the positive normalized poverty gaps generated by $\boldsymbol{x}$.
Definition 15 Given a weighting vector $\boldsymbol{w}=$ $\left(w_{1}, \ldots, w_{q}\right) \in[0,1]^{q}$ satisfying $w_{1} \geq \cdots \geq w_{q} \geq 0$ and $\sum_{i=1}^{q} w_{i}=1$, the function $A_{w}:[0,1]^{q} \longrightarrow[0,1]$ is defined as

$$
A_{w}\left(\boldsymbol{g}_{p}\right)=\sum_{i=1}^{q} w_{i} g_{\sigma(i)}
$$

where $\sigma$ is a permutation of $\{1, \ldots, q\}$ such that $g_{\sigma(1)} \geq \cdots \geq g_{\sigma(q)}$.

Notice that

$$
A_{w}\left(\boldsymbol{g}_{p}\right)=1-W_{w}\left(\frac{\boldsymbol{x}_{p}}{z}\right) .
$$

Aggregators in equation (4) can be considered as particular cases of OWA operators. Increasingness of $\left\{x_{i}\right\}$ guarantees decreasingness of $\left\{g_{i}\right\}$. And Sconcavity of $W_{w}$ implies S-convexity of $A_{w}$. These operators are stable for translations and scale invariant.

\subsection{The Sen poverty index and an alternative decomposition proposal}

We now introduce the Sen poverty index [30]. Although this is not Sen's original proposal, it is common to refer to this modified expression as the Sen index.

Definition 16 The Sen poverty index is the function $S:[0, \infty)^{n} \times(0, \infty) \longrightarrow[0,1]$ defined as

$$
S(\boldsymbol{x}, z)=\frac{2}{q n z} \sum_{i=1}^{q}\left(z-x_{(i)}\right)(q+0.5-i) .
$$

The summation structure of the $S$ index essentially combines the normalized gaps of the poor with $q$ positive coefficients which are larger for persons with larger income gaps: apart from the overall factor $2 / q n$, the largest gap has coefficient $q-0.5$ and the smallest gap has coefficient 0.5 , with decreasing unit step differences from one coefficient to the next. Actually, as we will see below, the $S$ index amounts to a convex combination of the normalized gaps, multiplied by the headcount ratio $q / n$. Moreover, the $S$ index satisfies PF, PM, TS, PS and RI.

Two alternative decompositions have been proposed of this index. On the one hand, Sen [30] shows that the index satisfies

$$
S(\boldsymbol{x}, z)=H(\boldsymbol{x}, z)\left(M(\boldsymbol{x}, z)+(1-M(\boldsymbol{x}, z)) G\left(\boldsymbol{x}_{p}\right)\right),
$$

where $G\left(\boldsymbol{x}_{p}\right)$ is the Gini index of the poor income.

$\mathrm{Xu}$ and Osberg [34] propose the following alternative decomposition

$$
S(\boldsymbol{x}, z)=H(\boldsymbol{x}, z)\left(M(\boldsymbol{x}, z)+M(\boldsymbol{x}, z) G\left(\boldsymbol{g}_{p}\right)\right),
$$

where $G\left(\boldsymbol{g}_{p}\right)$ is the Gini index of the poor gaps. 
However, as already mentioned, the choice between the Gini index of the poor income and the poor gaps is not innocuous. To illustrate this, let us consider two income distributions $\boldsymbol{x}^{1}=$ $(4,5,25,35)$ and $x^{2}=(3,4,22,32)$. Let us assume that the poverty line is $z=36$. Then, the corresponding poverty gap distributions are $\boldsymbol{g}^{1}=$ $\left(\frac{32}{36}, \frac{31}{36}, \frac{11}{36}, \frac{1}{36}\right)$ and $\boldsymbol{g}^{2}=\left(\frac{33}{36}, \frac{32}{36}, \frac{14}{36}, \frac{4}{36}\right)$. The Gini index of the income distributions concludes that the inequality among the poor is higher in the latter than in the former, $G\left(\boldsymbol{x}^{1}\right)=0.409<0.430=$ $G\left(\boldsymbol{x}^{2}\right)$. Nevertheless, this conclusion is reversed if the Gini index of the gap distributions is computed since $G\left(\boldsymbol{g}^{1}\right)=0.377>0.316=G\left(\boldsymbol{g}^{2}\right)$.

In what follows we propose an alternative decomposition of the Sen index that overcome this drawback.

The $S$ index can be rewritten as

$$
S(\boldsymbol{x}, z)=H(\boldsymbol{x}, z) \sum_{i=1}^{q} \frac{2(q-i)+1}{q^{2}} g_{\sigma(i)},
$$

where $\sigma$ is a permutation of $\{1, \ldots, n\}$ such that $g_{\sigma(1)} \geq \cdots \geq g_{\sigma(n)}$.

That is, an OWA operator $A_{G}:[0,1]^{q} \longrightarrow[0,1]$ applied to the poverty gaps, defined as

$$
A_{G}\left(\boldsymbol{g}_{p}\right)=\sum_{i=1}^{q} \frac{2(q-i)+1}{q^{2}} g_{\sigma(i)},
$$

is involved in the definition.

Proposition $6 A_{G}$ satisfies continuity, idempotency, symmetry, strict monotonicity, stability for translations, invariance for replications and $S$ convexity.

A straightforward application of the previous section allows us to compute the self-dual core and the anti-self-dual remainder of $A_{G}$. By Propositions 2 and 6 , the core $\widehat{A_{G}}$ is idempotent, symmetric, strictly monotonic, and stable for translations. The strictly monotonicity axiom implies that is increasing in the gap of a poor person. The stability for translations means that equal absolute changes in all poor gaps lead to the same absolute change in $\widehat{A_{G}}$. These properties can be regarded as basic properties of a poverty intensity index. In the particular case of the Sen index, Proposition 7 below shows that the core $\widehat{A_{G}}$ coincides with the aggregate income gap ratio, the archetypical measure of the poverty intensity.

Proposition $7 \widehat{A_{G}}\left(\boldsymbol{g}_{p}\right)=M(\boldsymbol{x}, z)$.

As regards the remainder, $\widetilde{A_{G}}$ is symmetric, fulfills that $\widetilde{A_{G}}\left(\boldsymbol{g}_{p}\right)=0$ if and only if $g_{1}=\cdots=g_{q}$, and Propositions 4 and 6 ensure that it is S-convex, and consequently the Pigou-Dalton transfer principle is satisfied. Hence, we can obtain a direct interpretation of $\widetilde{A_{G}}$ as a measure of inequality among the poor individuals. What is more interesting in our discussion, is that $\widetilde{A_{G}}$ is anti-self-dual, that is, inequality among the poor does not change if we focus on poverty gaps, or on achievements as measured by $\boldsymbol{x}_{p} / z$. This component is also invariant if the units in which income is measured change.

In addition, $\widetilde{A_{G}}$ is invariant for translations (Proposition 5), thus it measures inequality from an absolute point of view and remains invariant if the gaps of all the poor are increased by the same amount. Proposition 8 below shows that the antiself-dual core computed for the Sen index becomes the absolute Gini index of the poverty gaps, and equivalently, of the achievements normalized by the poverty line.

Proposition $8 \widetilde{A_{G}}\left(\boldsymbol{g}_{p}\right)=G_{A}\left(\frac{\boldsymbol{x}_{p}}{z}\right)$.

The next proposition provides a decomposition of the Sen index in three components: incidence, intensity and inequality. The interest of this result is that the inequality component measures equally the achievements and the gaps of the poor.

Proposition 9 The Sen index satisfies the following decomposition

$$
\begin{aligned}
& S(\boldsymbol{x}, z)=H(\boldsymbol{x}, z)\left(M(\boldsymbol{x}, z)+G_{A}\left(\boldsymbol{g}_{p}\right)\right)= \\
& H(\boldsymbol{x}, z)\left(M(\boldsymbol{x}, z)+G_{A}\left(\frac{\boldsymbol{x}_{p}}{z}\right)\right) .
\end{aligned}
$$

\section{References}

[1] Aristondo O, Lasso de la Vega C, Urrutia A. A new multiplicative decomposition for the Foster-Greer-Thorbecke poverty indices. Bulletin of Economic Research 2010; 62: 259-267.

[2] Atkinson AB. On the measurement of inequality. Journal of Economic Theory 1970; 2: 244263.

[3] Beliakov G, Pradera A, Calvo T. Aggregation Functions: A Guide for Practitioners. Heidelberg: Springer, 2007.

[4] Ben Porath E, Gilboa I. Linear measures, the Gini index and the income-equality tradeoff. Economic Theory 1994; 64: 443-467.

[5] Blackorby C, Donaldson D. Measures of relative equality and their meaning in terms of social welfare. Journal of Economic Theory 1978; 18: $59-80$.

[6] Calvo T, Kolesárova A, Komorníková M, Mesiar R. Aggregation operators: Properties, classes and construction methods. In: Calvo T, Mayor G, Mesiar R, editors. Aggregation Operators: New Trends and Applications. Heidelberg, Germany: Physica-Verlag; 2002, pp. 3104.

[7] Chakravarty SR. Inequality, Polarization and Poverty: Advances in Distributional Analysis. New York: Springer-Verlag; 2009. 
[8] Clark S, Hemming R, Ulph D. On indices for the measurement of poverty. Economic Journal 1981; 91: 515-526.

[9] Clarke PM, Gerdtham UG, Johannesson M, Bingefors K, Smith L. On the measurement of relative and absolute income-related health inequality. Social Science and Medicine 2002; 55: 1923-1928.

[10] Donaldson D, Weymark J. A single-parameter generalization of the Gini indices of inequality. Journal of Economic Theory 1980; 22: 67-86.

[11] Donaldson D, Weymark J. Ethically flexible Gini indices for income distributions in the continuum. Journal of Economic Theory 1983; 29: 353-358.

[12] Erreygers G. Can a single indicator measure both attainment and shortfall inequality? Journal of Health Economics 2008; 28: 885893.

[13] Fodor J, Roubens M. Fuzzy Preference Modelling and Multicriteria Decision Support. Dordrecht, The Netherlands: Kluwer Academic Publishers; 1994.

[14] Foster J, Greer J, Thorbecke E. A class of decomposable poverty measures. Econometrica 1984; 52: 761-766.

[15] García-Lapresta JL, Lasso de la Vega C, Marques Pereira RA, Urrutia AM. A class of poverty measures induced by the dual decomposition of aggregation functions. International Journal of Uncertainty, Fuzziness and Knowledge-Based Systems 2010; 18: 493-511.

[16] García-Lapresta JL, Llamazares B. Majority decisions based on difference of votes. Journal of Mathematical Economics 2001; 45: 463-481.

[17] García-Lapresta JL, Marques Pereira RA. The self-dual core and the anti-self-dual remainder of an aggregation operator. Fuzzy Sets and Systems 2008; 159: 47-62.

[18] Gini C. Variabilità e Mutabilità. Bologna: Tipografia di Paolo Cuppini; 1912.

[19] Grabisch M, Marichal JL, Mesiar R, Pap E. Aggregation Functions. Cambridge: Cambridge University Press; 2009.

[20] Jenkins S, Lambert P. Three 'I's of poverty curves and poverty dominance: TIPs for poverty analysis. Research on Economic Inequality 1998; 8: 39-56.

[21] Kakwani N. Inequality, Welfare and Poverty. In: Silber J, editor. Handbook of Income Inequality Measurement. Massachusetts: Kluwer Academic Publishers, 1999; pp. 599-628.

[22] Kolm SC. The optimal production of social justice. In Margolis J, Guitton H, editors. Publics Economics. London: Macmillan; 1969, pp. 145200.

[23] Lambert P, Zheng B. On the consistent measurement of achievement and shortfall inequality. Journal of Health Economics, forthcoming.

[24] Maes K, Saminger S, De Baets B. Represen- tation and construction of self-dual aggregation operators. European Journal of Operational Research 2007; 177: 472-487.

[25] Marshall AW, Olkin I. Inequalities: Theory of Majorization and Its Applications. Mathematics in Science and Engineering, vol 143. New York: Academic Press; 1979.

[26] Meharan F. Linear measures of income inequality. Econometrica 1976; 44: 405-409.

[27] Osberg L, Xu K. International comparisons of poverty intensity: Index decomposition and bootstrap inference. The Journal of Human Resources 2000; 35: 1-81.

[28] Quiggin J. Generalized expected utility theory: the rank-dependent model. Mathematical Social Sciences 1993; 1: 409-430.

[29] Sen AK. On Economic Inequality. Oxford: Oxford University Press; 1973.

[30] Sen AK. Poverty: An ordinal approach to measurement. Econometrica 1976; 44: 219-231.

[31] Shorrocks A. Revisiting the Sen poverty index. Econometrica 1995; 63: 1225-1230.

[32] Silber J. Handbook on Income Inequality. Kluwer Academic Press; 1999.

[33] Weymark J. Generalized Gini inequality indices. Mathematical Social Sciences 1981; 1: 409-430

[34] Xu K, Osberg L. The social welfare implications, decomposability, and geometry of the Sen family of poverty indices. Canadian Journal of Economics 2002; 35: 138-152.

[35] Yaari ME. The dual theory of choice under risk. Econometrica 1987; 55: 95-115.

[36] Yaari ME. A controversial proposal concerning inequality measurement. Journal of Economic Theory 1988; 44: 381-397.

[37] Yager RR. Ordered weighted averaging operators in multicriteria decision making. IEEE Transactions on Systems, Man and Cybernetics 1988; 8: 183-190.

[38] Yager RR, Kacprzyk J (eds.). The Ordered Weighted Averaging Operators: Theory and Applications. Norwell: Kluwer Academic Publishers; 1997.

[39] Yager RR, Kacprzyk J, Beliakov G (editors). Recent Developments in the Ordered Weighted Averaging Operators: Theory and Practice. Berlin: Springer-Verlag; 2011. 